\title{
Resultados preliminares del cultivo experimental de Gracilaria verrucosa (Hudson) Papenfuss (=G. caudata J. Agardh) (Rhodophyta: Gracilariaceae) en la Costa Caribe de Colombia
}

\author{
Jairo Napoleón Molina-Vargas, Ricardo Álvarez-León* \\ Fundación Verdes Horizontes, Manizales, Colombia
}

\begin{abstract}
Resumen
Se registran los resultados de la investigación realizada en la bahía de Fray Domingo, playa de Punta de Piedra, $\left(10^{\circ}\right.$ $52^{\prime} \mathrm{N}$ y $75^{\circ} 08^{\prime} \mathrm{O}$, aproximadamente), en el departamento de Atlántico, Caribe colombiano, entre el 30 de junio y el 1 de noviembre de 2004. Se llevó a cabo un ensayo de cultivo con el alga roja Gracilaria verrucosa utilizando una estructura artesanal cuya cuerda principal con mangas plásticas perforadas sirvió de soporte a las algas rojas; la estructura se colocó a una profundidad de $1,40 \mathrm{~m}$, fijándola al fondo y manteniéndola a flote. El tendido se revisó cada 15 días para observar el desarrollo de las ramas nuevas y el crecimiento apical de los filamentos. El crecimiento de las algas fue irregular, observándose que los penachos de algas fijadas originalmente crecieron uniformemente, en tanto que en los otros, solo se notó buen crecimiento en los extremos de las mangas. El peso fresco de la cuerda principal con mangas y algas fue de $2,5 \mathrm{~kg}$. Los filamentos más largos alcanzaron los $22 \mathrm{~cm}$. El crecimiento promedio de la fronda fue de $15 \mathrm{~cm}$. Las algas cultivadas crecieron sin ataques invasivos, aunque sí se observaron abundantes epífitas tanto en la cuerda principal como en las cuerdas secundarias de la estructura o módulo de cultivo, las cuales pertenecían, en orden de abundancia, a las clases Rhodophyceae, Phaeophyceae y Clorophyceae. La fronda de Hypnea musciformis fue la más abundante y la de mejor crecimiento; la fauna asociada estaba conformada especialmente por crustáceos juveniles: cangrejos, camarones y langostas.
\end{abstract}

Palabras clave: ficocultura, macroalgas rojas, Gracilaria verrucosa, Caribe, Colombia

Preliminary results of an experimental crop of Gracilaria verrucosa (Hudson) Papenfuss (= G. caudata J. Agardh) (Rhodophyta: Gracilariaceae) on the Colombian Caribbean Coast

\begin{abstract}
We present the results of the study carried out in Fray Domingo Bay, Punta de Piedra Beach $\left(10^{\circ} 52^{\prime} \mathrm{N}\right.$ and $75^{\circ} 08^{\prime}$ W approximately), Department of Atlántico, on the Colombian Caribbean coast, from June 30 to November 1, 2004. An experimental crop of the red algae Gracilaria verrucosa was developed using a handmade structure whose main rope with plastic perforated sleeves served as support for the algae. This structure was placed at a depth of $1.40 \mathrm{~m}$ and fixed to the bottom to maintain it floating. The structure was inspected every 15 days to observe the development of new branches and the apical growth in the filaments. The growth of the algae was irregular, as the feathers of those originally fixed grew evenly while in others growth was observed only in the end sections of the sleeves. The fresh weight of the main rope with sleeves and algae was $2.5 \mathrm{~kg}$. The longest filaments were $22 \mathrm{~cm}$ long. Fronds growth average was of $15 \mathrm{~cm}$. Cultivated algae grew without invasive attacks, but we did observe abundant epiphytes on the main and secondary ropes of the structure, which belonged to the Rhodophyceae, Phaeophyceae and Clorophyceae classes in order of abundance. Hypnea musciformis was the most abundant species and showed the best growth rate. The associated fauna consisted mainly of juvenile crustaceans: crabs, shrimps and lobsters.
\end{abstract}

Key words: Ficoculture, red macroalgae, Gracilaria verrucosa, Caribbean, Colombia

\section{Introducción}

La acuicultura en Colombia ha tenido un gran apoyo gubernamental y privado. La celebración de convenios y misiones ha permitido estructurar las bases científicas y tecnológicas para garantizar el manejo adecuado de sus recursos acuáticos; sin embargo, el desarrollo alcanzado no es el ideal para un país que, como Colombia, es megadiverso y cuenta con un considerable patrimonio renovable y no renovable, tanto marino como continental, en sus diferentes pisos térmicos y profundidades submarinas (Álvarez-León, 1982; Álvarez-León \& Rodríguez-Forero, 2000). 
El mito de "país subdesarrollado" que no cree en sí mismo, que no puede hacer más por falta de recursos y de tecnología, que está destinado a estar eternamente "en vías de desarrollo", es uno de los factores que inciden en el freno al desarrollo acuícola (Rodríguez-Gómez, et al., 1995; Álvarez-León \& Rodríguez-Forero, 2000). Además, la falta de difusión de los paquetes tecnológicos completos para especies nativas, la introducción de especies exóticas con paquetes tecnológicos transferidos (Hernández-Camacho, 1971; GutiérrezBonilla, 2002), así como la inadecuada canalización y fluidez de las líneas de crédito, la escasa publicación de resultados y la ausencia de publicidad para difundir los beneficios de los productos acuícolas han frenado o retardado el desarrollo del sector.

Aunque las especies utilizadas hasta el momento ascienden a 95 (algas, moluscos, crustáceos, peces), existe un enorme potencial para el desarrollo e implementación de biosistemas integrados en los ambientes costeros y continentales (Álvarez-León \& Rodríguez-Forero, 2000). En el caso de las algas marinas, el conocimiento de las especies nativas ha mejorado en cuanto a la composición específica en los diferentes ecosistemas y a su identificación. Estos esfuerzos se iniciaron con el trabajo de Bula-Meyer, 1989, en el que se resaltó el potencial ficológico y la factibilidad de los cultivos, pero fue sin duda el esfuerzo de Díaz-Pulido \& Díaz-Ruíz, 2003, el que proporcionó un panorama real de la riqueza y la diversidad de las algas en el Caribe colombiano, cuyo número asciende a 565 especies. El potencial químico de un número considerable de ellas ya ha sido evaluado (Álvarez-León, et al., 2007), así como la posibilidad de su utilización como fuente de coloides para la fabricación de medios de cultivo y extractos experimentales (Álvarez-León, et al., 2008).

El estudio de las algas exóticas, en cambio, apenas está comenzando con la única registrada hasta el momento, Eucheuma isiforme (C.Agardh) J.Agardh (que, según se comprobó, era en realidad Kappaphycus alvarezii), en La Guajira colombiana (Gallo-Peña \& Rincones-León, 2003; Rincones-León \& Gallo-Peña, 2004). Con motivo de la introducción al medio natural de dicha alga en el Cabo de la Vela, para apoyar las necesidades de la población pescadora de la tribu Wayúu, se realizó un informe detallado sobre la taxonomía, la biología y la ecología del alga (Díaz-Pulido \& Díaz-Ruíz, 2004). Recientemente, Rincones-León \& Moreno, 2009, presentaron los resultados de la experiencia desarrollada a través de un proyecto de maricultura de algas como alternativa de desarrollo sostenible de las comunidades costeras de La Guajira.

\footnotetext{
*Correspondencia:

Ricardo Álvarez-León, ricardoalvarezleon@gmail.com

Recibido: 27 de septiembre de 2013

Aceptado: 5 de mayo de 2014
}

\section{La ficocultura nacional}

Los cultivos experimentales de macroalgas bentónicas apenas comienzan en Colombia. El primer informe de investigación en este campo fue presentado por Bula-Meyer \& de Newball, 1983, quienes desarrollaron un cultivo experimental en el mar del alga roja Grateulopia filicina al sur del Rodadero en la costa del Caribe colombiano (Santa Marta, Magdalena).

Otros trabajos de investigación en el Caribe son los realizados por Morales-Sánchez \& Ángel-López, 1975, especialmente sobre Bryothamnion seaforthii y Gracilaria mammillaris en el Golfo de Morrosquillo, así como los de Gaviria-Melo, 1977, y González-Delgadillo \& Rojas-Ortega, 1995, llevados a cabo para conocer las características específicas de las algas encontradas y las épocas de su reproducción; el de Durán-Salcedo, 1979, sobre algunos aspectos ecológicos de Gracilaria cornea y su posible cultivo; el de Grajales-Orozco \& Poveda-Jiménez, 1997, sobre G. mammillaris y S. filiformis en Cartagena. También se han evaluado diferentes posibilidades de cultivo en los estudios de Montaña-Fernández, 2006, con Hypnea musciformis en las áreas de Taganga y Puerto Luz (Santa Marta), de Delgadillo-Garzón, et al., 2005, con Gracilaria spp. en la Guajira, y de Delgadillo-Garzón \& Newmark-Umbreit, 2008, con Grateloupia sp., Gracilaria cervicornis, Hydropuntia cornea e H. musciformis en la Bahía Portete y H. musciformis también en Taganga y Puerto Luz (Camacho-Hadad \& Montaña-Fernández, 2012).

En la actualidad se tiene información básica y hay ensayos preliminares de cultivo de nueve algas rojas en el Caribe, así como estudios preliminares básicos sobre dos algas rojas en el Pacífico (Tabla 1). La información sobre el cultivo de algas marinas en Colombia se puede resumir de acuerdo a las instituciones responsables así:

(1) La Universidad del Magdalena, a través de sus investigadores y con el apoyo de Colciencias, ha realizado diversos esfuerzos para evaluar la posible utilización industrial de las algas, así como de los cultivos que pudieran suplir la cantidad necesaria de materia prima, utilizando la rodófita G. filicina (Bula-Meyer \& de Newball, 1983; BulaMeyer, 1989a, 1989b).

(2) La Universidad de Bogotá Jorge Tadeo Lozano, a través de las tesis profesionales de sus egresados en Biología Marina y su participación en eventos, ha dado a conocer algunas características de los ecosistemas que han sido evaluados, al igual que las características específicas y las épocas de reproducción (Gaviria-Melo, 1977, Durán-Salcedo, 1979; González-Delgadillo \& Rojas-Ortega, 1995, DelgadilloGarzón, et al., 2005, Camacho-Hadad \& Sánchez, 2008), y organizó un seminario de investigación para evaluar las ventajas del alga asiática $K$. alvarezii, presente en algunas regiones del Caribe (García-Vásquez \& Pardo-Castro, 2002). 
(3) El Instituto para la Investigación de los Recursos Biológicos Alexander von Humboldt, a través de investigadores propios y contratados, ha comenzado a evaluar la factibilidad del cultivo de la rodófita nativa Eucheuma isiforme (Dawes, 1986; Littler \& Littler, 2000; Gallo-Peña \& Rincones-León, 2003), e introdujo $K$. alvarezii al medio natural (Díaz-Pulido \& Díaz-Ruíz, 2004) para ofrecer una alternativa económica a las comunidades Wayúu a lo largo del litoral de La Guajira en un sector de $25 \mathrm{~km}$ entre el Cabo de la Vela y Alema.

(4) Corpoguajira desarrolló el programa básico de capacitación de las comunidades del Cabo de la Vela, La Guajira, en el cultivo de algas marinas (Cuartas, 2004).

(5) La Universidad del Valle, a través de sus investigadores y de sus estudiantes de tesis en Biología, ha trabajado en detalle sobre aspectos ecológicos y fisiológicos de las algas del Pacífico, especialmente las asociadas al ecosistema de manglar Bostrychia calliptera y Catenella impudica. Asímismo, se han desarrollado trabajos sobre crecimiento, distribución y repoblamiento (Cárdenas-Cruz, 1992; Gómez \& Blanco, 1993; Peña-Salamanca, et al., 1996), así como del crecimiento en Caulerpasertularioides (MarínSalgado \& Peña-Salamanca, 2011).

\section{La ficocultura internacional}

El país con mayor experiencia en el cultivo artificial de Gracilaria es China. Los cultivos experimentales, y en algunos casos los de escala industrial, se vienen desarrollando en la costa sur del continente y en la costa este de Taiwán. Para el cultivo de Gracilaria se utilizan dos métodos: (1) la propagación vegetativa de las frondas en soportes artificiales, y (2) la diseminación de esporas sobre sustratos sólidos previamente acondicionados. La utilización de uno u otro método depende de las características biológicas de las especies. (Molina-Vargas, 2004).

El primer método es el más adecuado para las especies cuyas esporas son fáciles de obtener. Gracilaria tenuistipitata var. liui es un ejemplo, pues se propaga vegetativamente y puede cosecharse de tiempo en tiempo durante todo el periodo de crecimiento; Gracilaria blodgettii y Gracilaria parvispora son otros ejemplos de algas que se propagan a través de esporas liberadas de vez en cuando en primavera, verano y otoño. Con este método, las esporas se cultivan en estanques de agua de mar, en lagos salados o en salinas abandonadas. Los procedimientos son fáciles. El segundo método se usa para cultivar especies que tengan buena calidad y alto contenido de agar, tales como Gracilaria

Tabla 1. Relación de las algas nativas e introducidas cuyo cultivo ha sido evaluado en Colombia. A= Cultivo monoalgal, $B=$ Cultivo experimental, (*) especie asiática introducida. (1) Bula \& de Newball (1983), (2) Bula (1989b), (3) Camacho \& Sánchez (2008), (4) Camacho \& Sánchez (2012), (5) Delgadillo \& Newmark (2008), (6) Delgadillo-Garzón, et al. (2005), (7) Durán (1979), (8) García \& Pardo (2002), (9) Gaviria (1977), (10) Marín \& Peña (2011), (11) Montaña (2006), (12) Morales \& Ángel (1975), (13) Rincones \& Gallo (2004).

\begin{tabular}{|c|c|c|c|}
\hline Familias/Especies & Cuenca hidrográfica & Tipo de cultivo & Fuente (s) \\
\hline \multicolumn{4}{|l|}{ Chlorophyceae } \\
\hline 1. Chlorella sp. & Caribe & A & 9 \\
\hline \multicolumn{4}{|l|}{ Ulvophyceae } \\
\hline 2. Caulerpa sertularioides (J. V. Lamoroux) & Pacífico & A & 10 \\
\hline \multicolumn{4}{|l|}{ Thalassiosiraceae } \\
\hline 3. Skeletonema costatum & Caribe & A & 9 \\
\hline \multicolumn{4}{|l|}{ Myxophyceae } \\
\hline 4. Spirulina sp. & Caribe & A & 9 \\
\hline \multicolumn{4}{|l|}{ Rhodophyceae } \\
\hline \multicolumn{4}{|l|}{ Halymeniaceae } \\
\hline 5. Grateloupia filicina (J. V. Lamoroux) C. Agardh & Caribe & $\mathrm{B}$ & 1,2 \\
\hline \multicolumn{4}{|l|}{ Gracilariaceae } \\
\hline 6. Gracilaria cervicornis (Turner) J.Agardh & Caribe & B & 5 \\
\hline 7. Gracilaria mammillaris (Montagne) M.A.Howe & Caribe & B & 12 \\
\hline 8. Gracilarias pp. & & & 6 \\
\hline 9. Grateloupias pp. & & & 5 \\
\hline 10. Hydropuntia cornea (J. Agardh) M. J. Winne & Caribe & B & 5,7 \\
\hline 11. Hypnea musciformis (Wulfen) J. V. Lamouroux & Caribe & B & $3,4,5$ \\
\hline \multicolumn{4}{|l|}{ Solieriaceae } \\
\hline 12. Kappaphycus alvarez ii ( Doty) Doty ex P. C. Silva & Caribe* & B & 8,13 \\
\hline \multicolumn{4}{|l|}{ Rhodomelaceae } \\
\hline 13. Bryothamnion seaforth ii (Turner) Kützing & Caribe & B & 12 \\
\hline
\end{tabular}


asiatica, G. tenuistipitata, Gracilaria gigas y Gracilaria sjoestedtii, entre otras, las cuales se propagan solamente por medio de esporas (Molina-Vargas, 2004).

El cultivo artificial de Gracilaria se lleva a cabo teniendo en cuenta sus características biológicas. Se deben seleccionar sitios adecuados y tomar medidas efectivas con el fin de conseguir buenos resultados. El género Gracilaria es cosmopolita, está presente en mares de todas las latitudes, desde el Ártico hasta las regiones templadas y tropicales. Desde su primera descripción realizada por Greville en 1830, el registro de especies se ha venido incrementado y en la actualidad se han descrito 150 especies en diversas partes del mundo. Las algas del género Gracilaria exhiben talos erectos que surgen de un pequeño soporte en forma de disco. En la mayor parte de las especies los talos son cilíndricos, pero también se presentan especies con talos deprimidos o en forma de láminas. Son raras las especies con forma de hoja. Las ramas son alternas o, algunas veces, subdicotómicas (Molina-Vargas, 2004).

La estructura interna del talo de Gracilaria consiste de corteza y médula. Las células corticales son pequeñas. Una o dos de las capas más externas tienen células con los pigmentos característicos de la clase. La médula está constituida por grandes células parenquimatosas. Las capas de la corteza, el tamaño y el número de células medulares, así como el cambio de las células de la corteza a la médula se utilizan para la identificación de las especies (MolinaVargas, 2004).

A nivel internacional, Robledo-Ramírez, 2000, confirma que los resultados del cultivo de K. alvarezii (=Eucheuma cottonii =Eucheuma striatum var. tambalang) y de Eucheuma denticulatum (=E. spinosum) en el sur de Filipinas es el más espectacular y exitoso ejemplo de una empresa de maricultura a nivel mundial.

Se registran exitosos cultivos de G. verrucosa orientados a aprovechar económicamente sus coloides en las costas americanas del Pacífico chileno (Dellarossa, et al., 1980) y del mexicano (Lobo-Niembro et al., 1988), así como del Atlántico uruguayo (Fabiano \& Santana, 1996) y argentino (Boraso de Zaixo, 1987).

Las praderas de Gracilaria spp. en Latinoamérica y el Caribe varían entre la sobreexplotación y la ausencia de aprovechamiento, y Eucheuma spp. se consume en cantidades mínimas. El incremento en la producción de algas puede lograrse a través de un programa de selección de cepas a largo plazo que tome en cuenta la variabilidad intraespecífica, la variabilidad intraindividual $\mathrm{y}$, probablemente, diferencias entre fases nucleares (Espinoza-Avalos, 1996).

\section{Materiales y métodos}

\section{Selección de la playa para el cultivo}

La playa que se utilizó para el ensayo preliminar de cultivo está ubicada en la región de Punta de Piedra $\left(10^{\circ} 52^{\prime} 00^{\prime \prime}\right.$ $\mathrm{N}$ y $75^{\circ} 07^{\prime} 59^{\prime \prime} \mathrm{O}$, aproximadamente) en el departamento del Atlántico, Caribe colombiano. La playa se encuentra en la denominada bahía de Fray Domingo y tiene las siguientes características: parcialmente protegida de los vientos alisios por una barrera natural de pequeñas dunas y manglares (Rhizophora, Avicennia, Conocarpus); oleaje suave, profundidad aproximada de $1.20 \mathrm{~m}$ y fondo arenoso e inestable (Figura 1a, b, c, d).

El clima de la zona es de tipo tropical seco o xeromegaterno tropical, es decir, corresponde a una vegetación propia de la sequedad y las altas temperaturas. Conforme a la clasificación climática de Köppen, le corresponde un clima tropical de sabana. La temperatura promedio es de $27,4{ }^{\circ} \mathrm{C}$. De noviembre hasta principios de abril, o sea, coincidiendo con el invierno boreal, soplan los vientos alisios del noreste que mitigan en parte el intenso calor. Hacia fines de junio soplan los alisios del sureste, produciendo el veranillo de San Juan. El régimen de precipitación se rige por dos periodos: uno seco, de diciembre a abril, y otro lluvioso que abarca de abril a principios de diciembre. En abril o mayo empiezan las lluvias de "primera". Hacia fines de junio, gran parte de julio y a veces en agosto, tiende a disminuir la lluvia, constituyéndose una "seca" conocida como veranillo de San Juan. La precipitación anual promedio es de $821 \mathrm{~mm}$ (http://es.wikipedia.org/wiki/Barranquilla). En el agua, las variaciones suelen ser mínimas, entre 26,8 y $27,8^{\circ} \mathrm{C}$, el pH fluctúa entre 7,1 y 8,7 unidades y el oxígeno disuelto, entre 4,2 y 5,3 mg/1 (http://www.crautonoma.gov.co/documentos/ Planes/2013/4\%20Sintesis\%20Ambiental.pdf).

\section{Descripción del proceso de recolección y siembra}

Las frondas de G. verrucosa se recolectaron en la playa expuesta al mar abierto con oleaje de rompientes. El lecho marino en ese punto está constituido por rocas grandes mar adentro y rocas pequeñas cerca de la playa, de $30 \mathrm{~cm}$ de diámetro en promedio, en la zona eulitoral. Allí las rocas están cubiertas por arena y algas, y quedan expuestas según los movimientos del mar.

\section{Penachos frescos de G. verrucosa}

El 29 de junio de 2004, cuando se realizó la recolección, las rocas estaban cubiertas parcialmente por la arena y durante la marea baja las rocas con penachos de algas localizadas en la zona supralitoral quedaban al aire libre. Para la siembra se recogieron las algas ubicadas en la zona sublitoral a una profundidad de $70 \mathrm{~cm}$. No se hizo selección alguna para la recolección. Se desprendieron penachos grandes a mano y se llevaron a una caneca plástica (100 litros de capacidad 

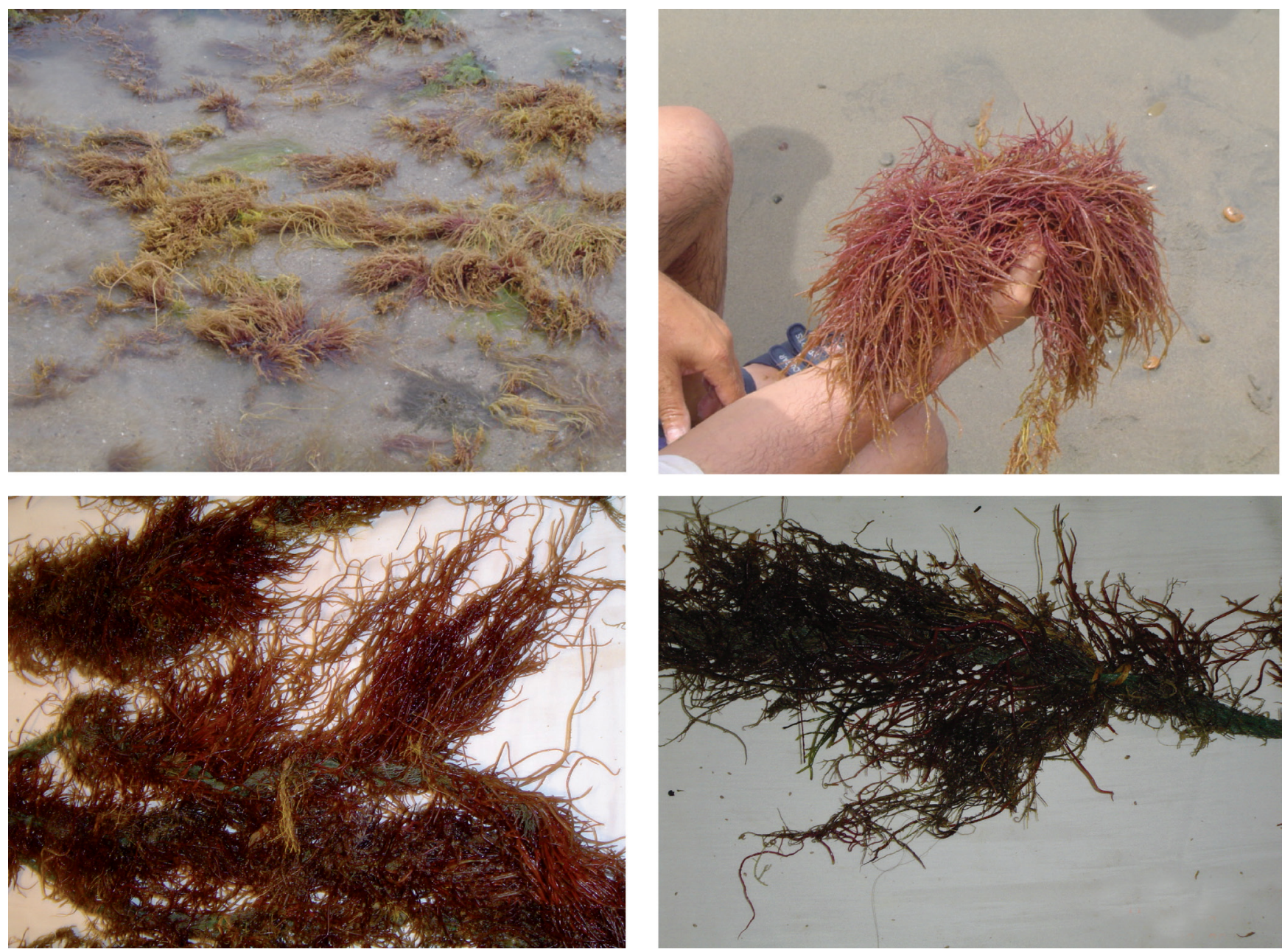

Figura 1. (a) Superior izquierda. Gracilaria verrucosa en su ambiente natural de arena y rocas subyacentes; durante la marea baja quedan expuestas al sol. (b) Superior derecha. Manojo fresco de G. verrucosa recolectado a $70 \mathrm{~cm}$ de profundidad; nótense los extremos amarillos de la fronda por exceso de luz solar. (c) Inferior izquierda. Penachos de G. verrucosa 120 días después de la siembra en mallas de plástico. (d) Inferior derecha. Frondas de Hypnea muciformis que crecieron asociadas a G. verrucosa.

aproximada) con agua de mar fresca. Se trasladó la caneca con las algas a la cabaña de trabajo, con el fin de montarlas para la siembra (Figura 1a, b).

\section{Enmallado y encordado de las frondas de Gracilaria verrucosa}

Para el entalegado se utilizaron mangas de malla plástica de las usadas para empacar frutas. Se cortaron piezas de $1 \mathrm{~m}$ (Figura 1b), se anudaron en un extremo y se llenaron y anudaron con atados de frondas maduras de $10 \mathrm{~cm}$. Los atados de frondas usados se cortaron cerca al disco basal. En cada manga se anudaron cinco atados; el peso de cada manga cargada de frondas fue de $50 \mathrm{~g}$. Las mangas se ataron a una cuerda principal de polietileno (diámetro: $1 / 4$ de pulgada). La cuerda principal pesó $200 \mathrm{~g}$ y el peso total fue de 1.200 g. Se ataron 20 mangas, a razón de una manga por metro.
La cuerda y las mangas con las frondas de G. verrucosa se colocaron dentro del tanque con agua de mar y se trasladaron a la orilla para la labor de siembra.

El 30 de junio de 2004 se instaló el ensayo de cultivo utilizando dos anclas fabricados artesanalmente con varillas de hierro de $1 / 2$ pulgada y tubos de PVC de 3 pulgadas de diámetro y $60 \mathrm{~cm}$ de largo. Se reforzó el anclaje con sacos de polipropileno cargados de arena hasta conseguir un peso de $15 \mathrm{~kg}$, aproximadamente. A las anclas se ataron cuerdas de nylon de $1 / 2 \mathrm{~cm}$ de diámetro y en el extremo libre se ataron envases desechables de plástico de $2 \frac{1}{2}$ litros de capacidad previamente sellados con sus tapas. Se tendió la cuerda principal con las mangas a una profundidad de $1.40 \mathrm{~m}$. Cada extremo de la cuerda se anudó a su respectiva cuerda de la boya. El tendido de 
la cuerda principal se dejó algo flojo y en el sentido de las corrientes naturales, de manera que las mangas tuvieran libertad de movimiento.

\section{Resultados}

El tendido se revisó cada 15 días y se observó el crecimiento de ramas nuevas y crecimiento apical de los filamentos. Algunas frondas (alrededor de $5 \%$ ) salieron de la malla y se perdieron.

El 1 de noviembre se retiró la cuerda principal con todas las mangas y las frondas. Se observó que el crecimiento de las algas fue irregular, es decir con longitudes diferentes. Algunas algas de los penachos entalegados crecieron uniformemente, en tanto que en otros solo se notó buen crecimiento en los extremos de la manga (Figura 1c).

El peso fresco de la cuerda principal con mangas y algas fue de $2,5 \mathrm{~kg}$. Los filamentos más largos alcanzaron los $22 \mathrm{~cm}$. El crecimiento promedio de las frondas fue de $15 \mathrm{~cm}$.

Contrario a lo esperado, las algas cultivadas crecieron sin ataques invasivos de moluscos y poliquetos, que sí se observaron en las algas epífitas y los crustáceos. Solo en algunos mechones se notó la presencia de H. musciformis, cuyas frondas alcanzaron el mismo tamaño de las de $G$. verrucosa, hacia el mes de octubre. Se observó mayor epifitismo sobre la cuerda principal, donde se fijaron

Tabla 2. Relación de las algas nativas asociadas al cultivo experimental de Gracilaria verrucosa en la bahía de Fray Domingo, Atlántico, Caribe colombiano. Los números corresponden a la colección de INVEMAR, donde fueron depositados los ejemplares.

\begin{tabular}{|c|c|}
\hline Especies & Número en el catálogo \\
\hline \multicolumn{2}{|l|}{ Chlorophyceae } \\
\hline \multicolumn{2}{|l|}{ Chaetomorphasp. } \\
\hline Cladophora prolifera (Roth) Kutzing & 157 \\
\hline \multicolumn{2}{|l|}{ Enteromorpha sp. } \\
\hline Ulva flexuosa Wulfen & 156 \\
\hline \multicolumn{2}{|l|}{ Phaeophyceae } \\
\hline \multicolumn{2}{|l|}{ Dictyota sp. } \\
\hline \multicolumn{2}{|l|}{ Dictyopteris sp. } \\
\hline Spatoglossum schroederi (C. Agardh) Kutzing & 133 \\
\hline \multicolumn{2}{|l|}{ Rhodophyceae } \\
\hline $\begin{array}{l}\text { Bryothamnion triquetrum (S. G. Gmelin) M. A. } \\
\text { Howe }\end{array}$ & 483,487 \\
\hline \multicolumn{2}{|l|}{ Gracilaria sp. } \\
\hline Gracilaria blodgettii Harvey & $482,484,485,486,488$ \\
\hline Hypnea musciformis (Wulfen) J. V. Lamouroux & 489 \\
\hline
\end{tabular}

abundantes frondas de $H$. musciformis, lo cual corrobora su preferencia por el sustrato de polietileno. Se fijaron, además, varias especies de Rhodophyceae (Bryothamnion, Gracilaria, Hypnea), Phaeophyceae (Dictyota, Dictyopteris, Spatoglossum) y Chlorophyceae (Chaetomorpha, Enteromorpha, Ulva). En el momento de retirar la cuerda se escaparon decenas de crustáceos juveniles: cangrejos, camarones y langostas, aunque algunos estaban aferrados a las algas, incluso después de que estas quedaron al aire libre (Figura 1c, d).

\section{Conclusiones}

En cinco meses el peso fresco de la cuerda principal con mangas y algas fue de $2,5 \mathrm{~kg}$. Los filamentos más largos alcanzaron los $22 \mathrm{~cm}$. El crecimiento promedio de las frondas fue de $15 \mathrm{~cm}$. Como en el resto del continente, su estabilidad se alcanzó entre los meses de junio, julio y agosto. Su crecimiento experimentó un continuo incremento y la densidad fue bastante estable. La tasa promedio de crecimiento se calculó en 1,08 en los 120 días de cultivo.

Las algas cultivadas crecieron sin ataques invasivos, aunque si se observó competencia por el espacio disponible en las cuerdas y mangas con otras algas (especies de Chlorophyceae, Phaeophyceae, Rhodophyceae).

La fauna acompañante incluyó, principalmente, crustáceos juveniles (cangrejos, camarones y langostas), por lo que se comprobó la importancia de las estructuras dispuestas en la bahía como refugio de los estadios juveniles de estos individuos.

Tabla 3. Relación de las algas nativas asociadas al sustrato rocoso cercano al cultivo experimental de Gracilaria verrucosa en la bahía de Fray Domingo, Atlántico, Caribe colombiano. Los números corresponden a la colección de INVEMAR, donde fueron depositados los ejemplares.

\begin{tabular}{lc}
\hline \multicolumn{1}{c}{ Especies } & Número en el catálogo \\
\hline Chlorophyceae & 157 \\
Cladophora prolifera (Roth) Kutzing & 156 \\
Ulva flexuosa Wulfen & 133 \\
Phaeophyceae & \\
$\begin{array}{l}\text { Spatoglossum schroederi (C. Agardh) Kutzing } \\
\text { Rhodophyceae }\end{array}$ & 483,487 \\
$\begin{array}{l}\text { Bryothamnion triquetrum (S. G. Gmelin) M. A. } \\
\text { Howe }\end{array}$ & \\
$\begin{array}{l}\text { Gracilaria } \text { sp. } \\
\text { Gracilaria blodgettii Harvey }\end{array}$ & 489 \\
\hline \begin{tabular}{l} 
Hypnea musciformis (Wulfen) J. V. Lamouroux \\
\hline
\end{tabular}
\end{tabular}


El éxito de proyectos con especies de importancia comercial ( $G$. verrucosa, entre otras) por ser fuentes de agar (Arias-Rojas \& Barragán-Pardo, 2005; DulceRivadeneira \& Montilla-Escudero, 2005; VillalobosRodríguez, et al., 2007; Montilla-Escudero, et al., 2011) y de otros compuestos asociados, permite incentivar la actividad del cultivo de organismos marinos por parte de las comunidades costeras y de los grupos académicos de investigación.

\section{Agradecimientos}

Especialmente al personal que colaboró en la recolección de las algas, en el montaje de la unidad de cultivo y en la retirada de la unidad en el momento de la recolección final y a la colega Marta Díaz-Ruíz, curadora de la colección de algas del INVEMAR, por la revisión de las algas acompañantes del cultivo y a las presentes en el sustrato rocoso adyacente al proyecto, y su determinación taxonómica.

\section{Conflicto de interés}

Los autores declaran que no tienen ningún conflicto de interés

\section{Bibliografía}

Álvarez-León, R., Rodríguez-Forero, A. 2000. La acuicultura en Colombia: estado actual y perspectivas. Rev. INFOPESCA Internacional. 6: 40-47.

Álvarez-León, R. 1982. Antecedentes y posibilidades para el desarrollo de la acuicultura en Colombia. Rev. Lat.-Amer. Acuic. 13: 9-19.

Álvarez-León, R, Pardo-Pardo, C. M. , Trespalacios-Rangel, A.A. 2007. Evaluación y utilización potencial de las macroalgas marinas del Caribe y el Pacífico de Colombia: estado actual de su conocimiento. UDC-Rev. BIOSALUD (Rev. Cienc. Básicas). 6: 113-129.

Álvarez-León, R., Trespalacios-Rangel A.A., Mercado-Reyes, M.M. 2008. Experiencias colombianas en la utilización de medios de cultivo y extractos experimentales a partir de macroalgas marinas del Caribe: estado actual de su conocimiento. UDC-Rev. BIOSALUD (Rev. Cienc. Básicas). 7: 107-113.

Arias-Rojas, M. del P., Barragán-Pardo, J. 2005. Determinación de la productividad del agar microbiológico a partir de algas rojas (Gracilaria cilindrica, G. mamillaris, G. verrucosa) con diferentes especies bacterianas. Tesis profesional. Facultad de Ingeniera Química. Univirsidad Nacional de Colombia

Boraso de Zaixo, A.L. 1987. Gracilaria verrucosa in Golfo Nuevo, Chubut, Argentina. Biological parameters and environmental factors. Hydrobiologica. 151/152: 239-244.

Bula-Meyer, G.A., de Newball, S. 1983. Cultivo experimental en el mar del alga béntica Grateloupia filicina (Cryptonemiales, Rhodophyta). Proyecto UTM/Colciencias, Santa Marta (Magdalena). Informe final, $45 \mathrm{p}$.
Bula-Meyer, G.A. 1989a. Las macroalgas bénticas marinas como recurso potencial económico en Colombia. Rev. Acad. Colomb. Cienc. 17 (65): 383-387.

Bula-Meyer, G.A. 1989b. Cultivo experimental en el mar del alga béntica Grateloupia filicina, pp. 101-104, In: de Oliveira, E. C. \& N. Kautsky (eds.) Workshop Cultivation of Seaweeds in Latin America. Sao Sebastiao (S. P.) Brazil. April 2-8, $141 \mathrm{p}$.

Camacho-Hadad, O.M., Sánchez, L. 2008. Dinámica temporal de biomasa y estados reproductivos de Hypnea musciformis en Punta La Loma, Magdalena: resultados preliminares. pp. 98, En: Garay, J. \& M. C. Páez (eds.). Seminario Nacional de Ciencia y Tecnología del Mar (SENALMAR). INVEMAR, San Andrés Isla, Colombia, 428 p.

Camacho-Hadad, O.M., Montaña-Fernández, J. 2012. Cultivo experimental en el mar del alga roja Hypnea musciformis en el área de Santa Marta, Caribe colombiano. Boletín de Investigaciones Marinas y Costeras, 41 (1): 29-46.

Cárdenas-Cruz, L.S. 1992. Estudio preliminar sobre el crecimiento y repoblamiento de dos especies de macroalgas rojas Catenella impudica y Bostrichia caliptera en condiciones naturales en Punta Soldado (Bahía de Buenaventura) costa Pacífica colombiana. Tesis Profesional. Facultad de Ciencias, Universidad del Valle.

Cuartas, A. 2004. Programa de capacitación en el cultivo de algas marinas a las comunidades del Cabo de la Vela, Península de La Guajira. Corpoguajira. Riohacha (Guajira). Informe final, $51 \mathrm{p}$.

Dawes, C. J. 1986. Botánica marina. 1ª Edición. Edit. LimusaWiley. México D. C. (México), 673 p.

Delgadillo-Garzón, O., Newmark-Umbreit, F. 2008. Cultivo piloto de microalgas rojas (Rhodophyta) en la Bahía Portete, Guajira colombiana. Bol. Inst. Inv. Mar. y Cost. 37 (2): 7-26

Delgadillo-Garzón, O., Díaz-Ruiz, M. Lara, O. 2005. Experimentos preliminares en Santa Marta para integrar a las macroalgas Gracilaria dentro de un policultivo en La Guajira, Caribe colombiano. pp. 56, En: Universidad de La Habana (ed.). VII Congreso de Ficología de Latinoamérica y el Caribe. V Reunión Iberoamericana de Ficología. Universidad de La Habana, La Habana (Cuba), $158 \mathrm{p}$.

Dellarossa, V., Romo, H., Alveal, K. 1980. Avances en el conocimiento ecológico de Gracilaria verrucosa en el área de Concepción, Chile. Bol. Inst. Oceanogr. 29 (2): 149-155.

Díaz-Pulido, G., Díaz-Ruíz, M.C. 2003. Diversity of benthic marine algae of the Colombian Atlantic, Biota Colombiana 4 (2): $203-246$

Díaz-Pulido, G., Díaz-Ruíz, M.C. 2004. Informe sobre la taxonomía, biología y ecología del alga cultivada en el Cabo de la Vela, penísula de La Guajira. INVEMAR-MHNM. Informe técnico, $55 \mathrm{p}$. 
Dulce-Rivadeneira, M., Montilla-Escudero, A. 2005. Obtención de agar microbiológico a partir de Gracilaria verrucosa: evaluación de su productividad en agar nutritivo con diferentes especies bacterianas. Tesis profesional. Facultad de Ciencias. Departamento de Microbiología. Pontificia Universidad Javeriana.

Durán-Salcedo, A. 1979. Algunos aspectos ecológicos de Gracilaria cornea J. Agardh (Gracilariaceae: Gigartinales: Rhodophyta). Tesis profesional. Facultad de Biología Marina. Universidad de Bogotá Jorge Tadeo Lozano, 30 p.

Espinoza-Avalos, J. 1996. Reflexiones sobre la cosecha, el cultivo y los coloides de las macroalgas marinas Gracilaria y Eucheuma en Latinoamérica y el Caribe. Interciencia. 21 (6): 255-258.

Fabiano, G., Santana, O. 1996. Cultivo de algas agaríferas (Gracilaria verrucosa) y abalón rojo (Haliotis rufescens) en Uruguay. Agrociencia: 222. http://www. fagro.edu.uy/ agrociencia/index.php/directorio/article/ viewFile/500/410

Gallo-Peña, H.M., Rincones-León, R.E. 2003. Factibilidad del cultivo de algas marinas. Proyecto Corpoguajira / IIRBAvH / FAO Fortalecimiento para el desarrollo de empresas rurales a partir de productos de la biodiversidad en el Cabo de la Vela, departamento de La Guajira. Consultoría Fase II. Bogotá D. C. (Colombia). Informe final, 55 p.

García-Vásquez, A., Pardo-Castro, PV. 2002. Observaciones de la adaptación de Kappaphycus alvarezii (Doty, 1988) y las variaciones de su tasa de crecimiento en el Acuario Mundo Marino. Seminario de Investigación. Facultad de Biología Marina, Universidad de Bogotá Jorge Tadeo Lozano. Santa Marta (Magdalena).

Gaviria-Melo, S. 1977. Observación de los períodos de reproducción de algunas especies de algas colombianas. Tesis profesional. Facultad de Biología Marina, Universidad de Bogotá Jorge Tadeo Lozano.

Gómez, S., Blanco, M.C. 1993. Tasas de crecimiento en dos especies de algas rojas de importancia comercial en la bahía de Buenaventura. Tesis profesional. Facultad de Ciencias, Universidad del Valle.

González-Delgadillo, A. M., Rojas-Ortega, J. 1995. Estructura y composición de la comunidad macroalgal en el sistema arrecifal coralino del archipiélago de San Bernardo, Caribe colombiano. Tesis profesional. Facultad de Biología Marina, Universidad de Bogotá Jorge Tadeo Lozano, 67 p.

Grajales-Orozco, A., Poveda-Jiménez, L.E. 1997. Abundancia, estados reproductivos y algunos parámetros químicos de las macroalgas Gracilaria mammilaris y Solieria filiformis en Cartagena de Indias, Caribe colombiano. Tesis profesional. Facultad de Biología Marina. Universidad de Bogotá Jorge Tadeo Lozano.

Gutiérrez-Bonilla, F. de P. 2002. Introducción, trasplante y repoblación con recursos hidrobiológicos: marco normativo nacional, internacional y competencias institucionales en
Colombia. MMA/RAMSAR/CVC. Santa Fe de Bogotá D. C. (Colombia), 24 p.

Hernández-Camacho, J. I. 1971. Aspectos sobre la introducción de especies exóticas. I Sem. Nal. de Piscicultura. Manizales (Caldas), enero 12-16.

Littler, D. C., Littler M.M. 2000. Caribbean reef plants. Offshore Graphics. Washington (USA), 542 p.

Lobo-Niembro, J.M., Marcos Ramírez, R. 1988. Cobertura, fenología y gel de Gracilaria verrucosa (Hudson) Papenfuss (Rhodophyta, Gigartinales) durante un ciclo anual en el estero de Punta Banda, Baja California. Tesis profesional (Oceanólogo), Facultad de Ciencias Marinas, Universidad Autónoma de Baja California, 37 p.

Marín-Salgado, H. \& E. Peña-Salamanca. 2011. Ensayos para el cultivo experimental de Caulerpasertularioides (Chlorophyta, Bryopsidales) en estanques de camarón en el Pacifico colombiano. Rev. El Bohío (Por un Medio Ambiente Responsable), 1 (3): 1-9.

Molina-Vargas, J.N. (trad.). 2004. Manual de entrenamiento para el cultivo de Gracilaria spp. y el procesamiento de algas en China. FAO. (Traducción Jairo Napoleón Molina-Vargas, 2004) (Revisión técnica Ricardo Álvarez-León, 2005).

Morales-Sánchez, J.E., Ángel-López, L. 1975. Observaciones ecológicas en algas marinas en Punta San José, Golfo de Morrosquillo, costa Caribe colombiana. Tesis profesional, Facultad De Ciencias. Universidad Nacional de Colombia.

Montaña-Fernández, J. 2006. Ensayo de cultivo en medio natural de la microalga Hypnea musciformis (Wulfen) Lamouroux en las áreas de Taganga y Puerto Luz (Santa Marta). Tesis profesional. Facultad de Biología Marina, Universidad de Bogotá Jorge Tadeo Lozano, 75 p.

Montilla-Escudero, E.A., Dulce-Rivadeneira, M.F., QuevedoHidalgo, B.E., Mercado-Reyes, M., Álvarez-León, R., Molina-Vargas, J.N., Trespalacios-Rangel, A.A. 2011. Efecto del tratamiento alcalino sobre la productividad y las propiedades del agar-agar proveniente de Gracilaria verrucosa. INVEMAR-Bol. Invest. Mar. y Cost. 40 (1): $75-88$.

Peña-Salamanca, E.J., Álvarez-León, R. 2006. Experiencias en el cultivo experimental de algas rojas en el Caribe y Pacífico de Colombia. UDC- Luna Azul. (Rev. Cientif. - Versión en línea), 23: 1-5.

Peña-Salamanca, E.J., Cárdenas, E., Bolívar, G. 1996. Growth and distribution of two algae in a tropical estuary in the Pacific coast of Colombia, S. A. UBJTL-Bol. Ecotrópica. 39: $177-188$.

Rincones-León, R.E. \& H. Gallo-Peña. 2004. Proyecto Jimoula: el cultivo de algas marinas como alternativa sustentable para las comunidades costeras de la Península de la Guajira, BIOTACOL Ltda.. Riohacha (Guajira), 11 p.

Rincones-León, R.E., Moreno, D.A. 2009. La maricultura de algas como alternativa de desarrollo sostenible de las comunidades 
costeras de La Guajira, En: Álvarez-León, R. (ed.) Memorias Resúmenes del Congreso Internacional sobre las Experiencias de la Iniciativa ZERI en Colombia. Manizales (Caldas) Colombia, 19 a 20 de noviembre de 2009.

Robledo-Ramírez, D. 2000. Agronomía marina: una alternativa para el desarrollo de las comunidades pesqueras de Dzilam de Bravo. Proy. M151 INP-CIEA. Departamento de Recursos del Mar-Laboratorio de Ficología. Mérida (Yucatán) México. Informe final, 49 p.

Rodríguez-Gómez, H., Polo-Romero, G., Mora-Lara, C.O. (eds.). 1995. Fundamentos de acuicultura marina. Ministerio de Agricultura y Desarrollo Rural / INPA. Santafé de Bogotá D.C. (Colombia), 225p.

Villalobos-Rodríguez, A. P., Calderón-Carvajal, D.L., Figueroa, C., Fierro-Mora, J., Otálora-Pinzón, G., Álvarez-León, R., Quevedo-Hidalgo, B., Mercado-Reyes, M. M., Huertas-Valero, M. G., Trespalacios-Rangel, A. A. 2007. Evaluación por el método ecométrico del agar obtenido de algas rojas colombianas. PUJ-Universitas Scientiarum. 12 (Edic. Esp. III): 57-65. 